\title{
Partially coherent fields with circular coherence
}

\author{
M. Santarsiero ${ }^{1}$, R. Martínez-Herrero ${ }^{2}$, D. Maluenda ${ }^{2}$, J. C. G. de Sande ${ }^{3}$, G. Piquero², \\ AND F. GORI ${ }^{1}$ \\ ${ }^{1}$ Dipartimento di Ingegneria, Università Roma Tre, Via V. Volterra 62, 00146 Rome, Italy \\ ${ }^{2}$ Departamento de Óptica, Universidad Complutense de Madrid, Ciudad Universitaria, 28040 Madrid, Spain \\ ${ }^{3}$ ETSIS de Telecomunicación, Universidad Politécnica de Madrid, Campus Sur 28031 Madrid, Spain \\ *Corresponding author: email@my-email.com
}

Compiled February 15, 2017

A new class of partially coherent light sources is introduced. At the source plane, they exhibit perfect coherence along any annulus that is concentric to the source center. Between two points at different distances from the center, coherence can be partial or even vanishing. Such sources can be synthesized by using a generalized form of van Cittert-Zernike theorem where axial sources are used. Beam radiated by this type of sources are analyzed at the source plane and upon free propagation for some simple cases. () 2017 Optical Society of America

OCIS codes: (030.1640) Coherence; (030.6600) Statistical optics.

http://dx.doi.org/10.1364/ao.XX.XXXXXX

Modeling partially coherent sources with different characteristics across the source plane and on propagation has been a big deal during the last decades [1-21]. Experimental procedures aimed at synthesizing sources with tunable coherence properties have been also presented [22-27]. For a detailed review on the structure and synthesis of partially coherent sources, see [28] and references therein. Among the conceivable partially coherent sources, those of the Schell-model type [29] have played a crucial role in coherence theory. Such sources are characterized by a shift-invariant spectral degree of coherence [29] and then provide a useful model to represent many natural sources. Schell-model sources can be synthesized in a rather simple way, because they can be produced starting from a primary incoherent two-dimensional source and exploiting the van Cittert-Zernike theorem [29].

In this letter we present a class of planar, circularly symmetric, partially coherent sources, for which the degree of coherence between two points depends only on their distances from the source center. This means, in particular, that source regions containing points with high correlation have the shape of a donut and, in particular, the fields at any pair of points lying along a ring concentric with the source are mutually perfectly coherent. Natural sources endowed with such a property are rather unusual but, as will be shown, they can be synthesized in a very simple way. On the other hand, their propagation properties can be of interest, because their symmetry exactly reflects that of most optical systems used in practical applications.
The proposed class of sources is characterized by a crossspectral density (CSD) at the source plane of the type

$$
W_{0}\left(r_{1}, r_{2}\right)=\tau^{*}\left(r_{1}\right) \tau\left(r_{2}\right) g\left(\frac{r_{2}^{2}-r_{1}^{2}}{\delta_{\mu}^{2}}\right),
$$

where $r$ is the modulus of the position vector on the source plane $(\boldsymbol{r})$, while $g$ and $\tau$ are complex-valued functions. The parameter $\delta_{\mu}$ has been introduced as a positive quantity having dimensions of a length, in order for $g$ to be a function of a dimensionless argument. As we shall see, $\delta_{\mu}$ can be related to the coherence properties of the source. Note that CSDs written as in Eq. (1) fulfill the non-negativeness condition, and therefore represents physically realizable sources, for any $g$ having non-negative Fourier transform [29]. Here and in the following we shall omit the explicit dependence from the temporal frequency.

The irradiance profile of the source, namely, $I_{0}(r)=W_{0}(r, r)$, is completely determined by the function $\tau(r)$, while the degree of spectral coherence between the points $r_{1}$ and $r_{2}$, defined as [29]

$$
\mu_{0}\left(r_{1}, r_{2}\right)=\frac{W_{0}\left(r_{1}, r_{2}\right)}{\sqrt{W_{0}\left(r_{1}, r_{1}\right) W_{0}\left(r_{2}, r_{2}\right)}},
$$

is related to the function $g$. More precisely, it turns out that

$$
\mu_{0}\left(r_{1}, r_{2}\right)=\frac{\tau^{*}\left(r_{1}\right) \tau\left(r_{2}\right)}{\left|\tau\left(r_{1}\right) \tau\left(r_{2}\right)\right|} g\left(\frac{r_{2}^{2}-r_{1}^{2}}{\delta_{\mu}^{2}}\right),
$$

where, without loss of generality, we set $g(0)=1$. In particular, the modulus of the degree of coherence depends only on the difference between the squared radial coordinates of the two points, and is one for any pairs of points lying on a circle centered on the axis origin. The analytical form of such a CSD reminds that of a source of the Schell-model type, but in the present case the dependence of the modulus of the degree of coherence is through the difference $r_{2}^{2}-r_{1}^{2}$, instead that through the difference of the position vectors themselves $\left(\boldsymbol{r}_{2}-\boldsymbol{r}_{1}\right)$.

To illustrate some general coherence properties of this kind of sources, we first consider the case where one of the two points coincides with the axis origin. Then, the parameter $\delta_{\mu}$ can be defined in such a way to represent the maximum distance from the origin for which a significant correlation exists between the field values at the two points. In this way, the quantity $\pi \delta_{\mu}^{2}$ provides an estimate of the coherence area of the source. In a 
more general case, the argument of the function $g$ can be written as

$$
\frac{r_{2}^{2}-r_{1}^{2}}{\delta_{\mu}^{2}}=\frac{\left(r_{2}+r_{1}\right)\left(r_{2}-r_{1}\right)}{\delta_{\mu}^{2}}=\frac{2 \bar{r}}{\delta_{\mu}^{2}}\left(r_{2}-r_{1}\right),
$$

where $\bar{r}=\left(r_{1}+r_{2}\right) / 2$ is the average radial distance of the two points. Equation (4) shows that, if $\bar{r}$ is kept fixed, the dependence of the degree of coherence on the radial coordinates of the two points is just of the Schell-model type in one dimension. Therefore, for any fixed value of $\bar{r}$ we can identify an annular region, having mean radius $\bar{r}$ and width $\delta_{\mu}^{2} / 2 \bar{r}$, within which a significant correlation exists between the field values ( $a$ "coherence donut"). The width of this annulus decreases on increasing $\bar{r}$ but its area, approximately given by $2 \pi \bar{r} \delta_{\mu}^{2} / 2 \bar{r}=\pi \delta_{\mu}^{2}$, remains constant. In this sense, $\pi \delta_{\mu}^{2}$ can be taken as a measure of the coherence area across the source. Plots describing the behavior of the degree of coherence of the source are shown in Fig. 1 for the case $g(\cdot)=\operatorname{sinc}(\cdot)$, with $\delta_{\mu}=1$ (in arbitrary units).

The coherence properties of the radiated field across a transverse plane at a distance $z$ from the source can be obtained, under paraxial approximation, by means of the Fresnel diffraction integral. The resulting CSD is [29]

$$
\begin{aligned}
& W\left(\mathbf{R}_{1}, \mathbf{R}_{2}, z\right)=\frac{1}{\lambda^{2} z^{2}} \iint W_{0}\left(r_{1}, r_{2}\right) \\
& \quad \times \exp \left[-\frac{\mathrm{i} k}{2 z}\left(\left|\mathbf{R}_{1}-\mathbf{r}_{1}\right|^{2}-\left|\mathbf{R}_{2}-\mathbf{r}_{2}\right|^{2}\right)\right] \mathrm{d} \mathbf{r}_{1} \mathrm{~d} \mathbf{r}_{2},
\end{aligned}
$$

where $\mathbf{R}_{1}$ and $\mathbf{R}_{2}$ are the position vectors of two points across the plane $z=$ constant. Taking the circular symmetry of $W_{0}$ into account, Eq. (5) can be written as

$$
\begin{gathered}
W\left(\mathbf{R}_{1}, \mathbf{R}_{2}, z\right)=\frac{k^{2}}{z^{2}} \exp \left[\frac{\mathrm{i} k}{2 z}\left(R_{2}{ }^{2}-R_{1}{ }^{2}\right)\right] \\
\times \int_{0}^{\infty} \int_{0}^{\infty} \tau^{*}\left(r_{1}\right) \tau\left(r_{2}\right) g\left(\frac{r_{2}^{2}-r_{1}^{2}}{\delta_{\mu}^{2}}\right) \exp \left[\frac{\mathrm{i} k}{2 z}\left(r_{2}{ }^{2}-r_{1}{ }^{2}\right)\right] \\
\times J_{0}\left(\frac{k R_{1}}{z} r_{1}\right) J_{0}\left(\frac{k R_{2}}{z} r_{2}\right) r_{1} r_{2} \mathrm{~d} r_{1} \mathrm{~d} r_{2}
\end{gathered}
$$

where $k$ is the wave number and $J_{0}(\cdot)$ is the Bessel function of the first kind and zero order [30]. Of course, the CSD preserves its circular symmetry during propagation.

Besides the trivial case $\delta_{\mu} \rightarrow \infty$, which gives rise to a perfectly coherent beam, the opposite limiting case is of interest. When $\delta_{\mu} \rightarrow 0$, in fact, the width of the coherence donuts tends to zero but perfect correlation still exists between points lying on concentric rings. Therefore, the source consists of the superposition of a continuous set of mutually incoherent but perfectly coherent thin annular sources. Each such coherent source gives rise to a zero-order Bessel beam [31], with its own width and amplitude, so that the propagated CSD reduces to the sum of CSDs of perfectly coherent Bessel beams.

The expression of the CSD in the far zone is directly obtained from Eq. (6) on neglecting the exponential term inside the integral. To give an example, we take $g$ as a sinc function (with $\delta_{\mu}=1$, in arbitrary units), and the irradiance profile as a Gaussian, i.e., $\tau(r)=\exp \left(-a r^{2}\right)$, with $a$ being related to the width of the source irradiance. Figure 2 shows the behavior of the spectral degree of coherence across a transverse plane in the far zone. Normalized variables $R_{1} / z$ and $R_{2} / z$ are used.

In the following of the letter we present a possible experimental setup aimed at producing sources of the type introduced here. About this, it is worth doing a remark on the analytical
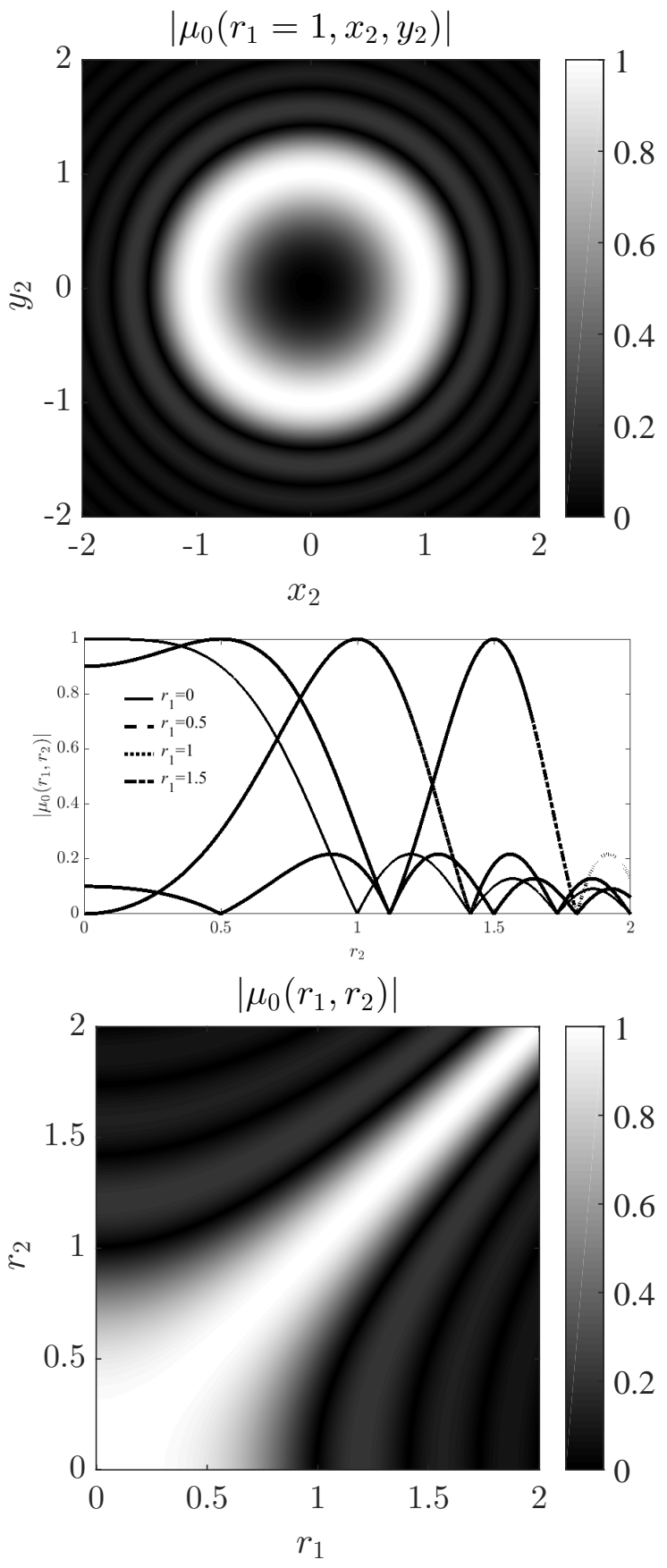

Fig. 1. Absolute value of the spectral degree of coherence, with $g(\cdot)=\operatorname{sinc}(\cdot)$ : across the source plane for $r_{1}=1$ (upper plot); as a function of $r_{2}$ for several values of $r_{1}$ (central plot); across the plane $r_{1}, r_{2}$ (lower plot). All lengths are normalized to the value of $\delta_{\mu}$ 
structure of the CSD in Eq. (1). Note, in fact, that the position of a typical source point enters only through a single, scalar variable. Accordingly, the source itself can be thought of, in a well defined sense, as one-dimensional. As we shall see, this remark is a key for suggesting practical realization schemes. A possible scheme
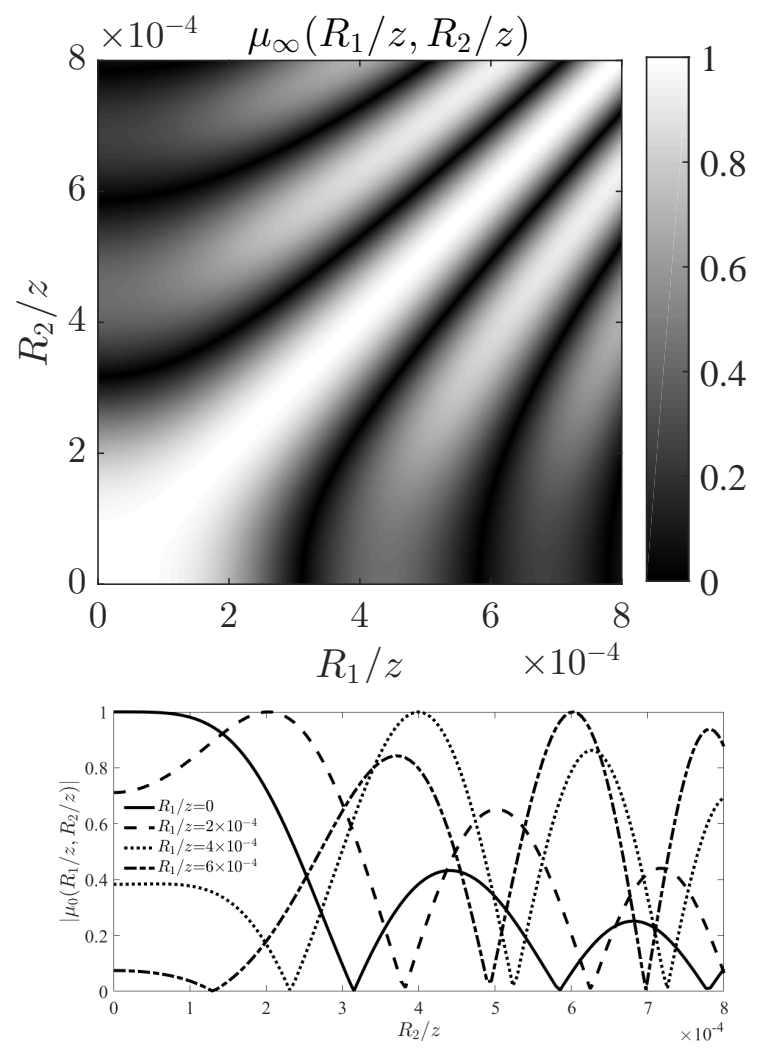

Fig. 2. Absolute value of the spectral degree of coherence across a transverse plane in the far zone, with $g(\cdot)=\operatorname{sinc}(\cdot)$, $\tau(r)=\exp \left(-a r^{2}\right)$, and $a=1$ (in units of $\delta_{\mu}$ ): across the plane $\left(R_{1} / z, R_{2} / z\right)$ (upper plot) and as a function of $R_{2} / z$ for several values of $R_{1} / z$ (lower plot).

is shown in Fig. 3. The source plane is located across the image focal plane of a converging lens having focal length $f$. Behind the lens, a line source is placed along the $z$ axis. To denote the line-source elements it is convenient to introduce an axis, parallel to the $z$ axis but oriented in the opposite direction, and having its origin at the lens position. The coordinate on such axis is denoted by $\xi$. At the plane $z=0$ a thin planar transparency, or a spatial light modulator, is placed, having transmission function $\tau(r)$.

Each element of the source, of length $\mathrm{d} \xi$, radiates a spherical wave, whose amplitude is proportional to $A(\xi) \mathrm{d} \xi$, where $A(\xi)$ is an amplitude density. Such spherical wave passes through the lens and, eventually, through the transparency. We are going to evaluate the CSD of the field across the plane $z=0$, just beyond the transparency. We will assume the paraxial conditions to be satisfied.

Starting from the Fresnel propagation formula, it is easy to evaluate the field produced across the plane $z=0$ by a single source element as

$$
\mathrm{d} V_{0}(r)=\frac{-\mathrm{i}}{\lambda f} A(\xi) e^{\mathrm{i} k(\xi+f)} e^{\frac{\mathrm{i} k}{2 f}\left(1-\frac{\xi}{f}\right) r^{2}} \mathrm{~d} \xi
$$

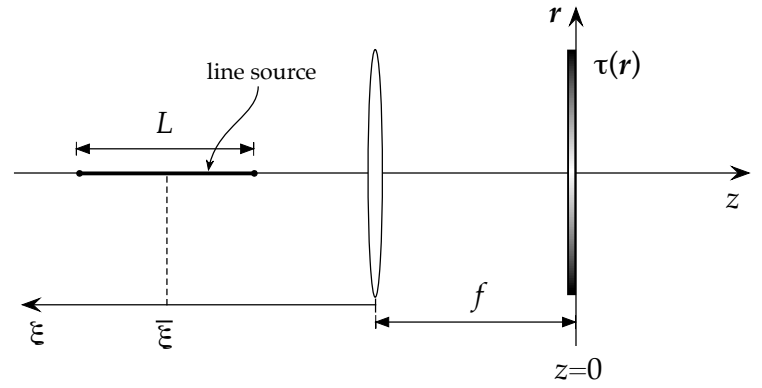

Fig. 3. Basic experimental scheme

where $\lambda=2 \pi / k$, and the field produced by the whole line source after the transparency turns out to be

$$
V_{0}(r)=\tau(r) \frac{-\mathrm{i}}{\lambda f} \int_{-\infty}^{\infty} A(\xi) e^{\mathrm{i} k(\xi+f)} e^{\frac{\mathrm{i} k}{2 f}\left(1-\frac{\xi}{f}\right) r^{2}} \mathrm{~d} \xi .
$$

Although the source length is generally finite, the integral in Eq. (7) has been formally extended between $-\infty$ and $\infty$, the real extension of the segment being considered through the function $A(\xi)$.

The CSD of the field beyond the transparency can be evaluated as

$$
W_{0}\left(r_{1}, r_{2}\right)=\left\langle V_{0}^{*}\left(r_{1}\right) V_{0}\left(r_{2}\right)\right\rangle,
$$

where the angle brackets denote ensemble average. On inserting from Eq. (8) into Eq. (9) we readily obtain

$$
\begin{aligned}
& W_{0}\left(r_{1}, r_{2}\right)=\frac{1}{(\lambda f)^{2}} \tau^{*}\left(r_{1}\right) \tau\left(r_{2}\right) e^{\frac{\mathrm{i} k}{2 f}\left(r_{2}^{2}-r_{1}^{2}\right)} \\
& \times \int_{-\infty}^{\infty} \int_{-\infty}^{\infty} W_{\mathrm{S}}\left(\xi_{1}, \xi_{2}\right) e^{\mathrm{i} k\left(\xi_{2}-\xi_{1}\right)} e^{-\frac{\mathrm{i} k}{2 f^{2}}\left(r_{2}^{2} \xi_{2}-r_{1}^{2} \xi_{1}\right)} \mathrm{d} \xi_{1} \mathrm{~d} \xi_{2},
\end{aligned}
$$

where the correlation function of the field along the source, namely,

$$
W_{\mathrm{S}}\left(\xi_{1}, \xi_{2}\right)=\left\langle A^{*}\left(\xi_{1}\right) A\left(\xi_{2}\right)\right\rangle,
$$

has been introduced.

Equation (10) takes a simpler form if the points of the line source are supposed to radiate independently from one another. In such a case the line source is spatially incoherent and its CSD can be given the form

$$
W_{\mathrm{S}}\left(\xi_{1}, \xi_{2}\right)=I_{\mathrm{S}}\left(\xi_{1}\right) \delta\left(\xi_{1}-\xi_{2}\right),
$$

where $I_{\mathrm{S}}(\xi)$ is proportional to the irradiance along the line source and $\delta(\cdot)$ is the Dirac delta function. Therefore, the expression of the CSD of the source across $z=0$ becomes

$$
\begin{aligned}
W_{0}\left(r_{1}, r_{2}\right)= & \frac{1}{(\lambda f)^{2}} \tau^{*}\left(r_{1}\right) \tau\left(r_{2}\right) e^{\frac{\mathrm{i} k}{2 f}\left(r_{2}^{2}-r_{1}^{2}\right)} \\
& \times \int_{-\infty}^{\infty} I_{\mathrm{S}}(\xi) e^{-2 \pi \mathrm{i}\left(\frac{r_{2}^{2}-r_{1}^{2}}{2 \lambda f^{2}}\right) \xi} \mathrm{d} \xi .
\end{aligned}
$$

It has to be noted that the function depending on $r_{2}^{2}-r_{1}^{2}$ in the latter equation, except for a phase factor giving account for a spherical wavefront, is given by the one-dimensional Fourier 
transform of the intensity of the line source. Therefore, Eq. (13) expresses an extension of van Cittert-Zernike theorem [29] for axial line sources.

Let us now go on from Eq. (13) and consider the realistic case of a finite line source. We denote by $L$ the length of the source and by $\bar{\xi}$ the coordinate of its central point. Then, we change the integration variable $\xi$ into $\zeta=\xi-\bar{\xi}$ in such a way that the integration interval becomes symmetric and, to simplify the notations, we introduce the function $G(\zeta)=I_{S}(\bar{\xi}+\zeta)$. Equation (13) then becomes

$$
\begin{aligned}
W_{0}\left(r_{1}, r_{2}\right)= & \frac{1}{(\lambda f)^{2}} \tau^{*}\left(r_{1}\right) \tau\left(r_{2}\right) e^{\frac{\mathrm{i} k}{2 f}\left(1-\frac{\bar{\xi}}{f}\right)\left(r_{2}^{2}-r_{1}^{2}\right)} \\
& \times \int_{-L / 2}^{L / 2} G(\zeta) e^{-2 \pi \mathrm{i}\left(\frac{r_{2}^{2}-r_{1}^{2}}{2 \lambda f^{2}}\right) \zeta} \mathrm{d} \zeta
\end{aligned}
$$

The exponential term outside the integral accounts for an overall spherical curvature and vanishes if we let the central point of the line source to coincide with the focus of the lens (i.e., if we put $\bar{\xi}=f$ ). With such a position, Eq. (14) takes the form

$$
W_{0}\left(r_{1}, r_{2}\right)=\frac{1}{(\lambda f)^{2}} \tau^{*}\left(r_{1}\right) \tau\left(r_{2}\right) \tilde{G}\left(\frac{r_{2}^{2}-r_{1}^{2}}{2 \lambda f^{2}}\right),
$$

which coincides with Eq. (1) with

$$
g\left(\frac{r_{2}^{2}-r_{1}^{2}}{\delta_{\mu}^{2}}\right)=\frac{1}{(\lambda f)^{2}} \tilde{G}\left(\frac{r_{2}^{2}-r_{1}^{2}}{2 \lambda f^{2}}\right)
$$

We finally stress that any bona fide CSD of the type in Eq. (1) can be synthesized using the proposed experimental scheme. The irradiance along the line source has to be chosen as the Fourier transform of the function $g$. Conversely, any Fourier transformable intensity $I_{S}(\xi)$ gives rise to a new source described by a CSD of the type in Eq. (1). It is much the same that happens when two-dimensional Schell-model sources are synthesized starting from planar spatially incoherent sources [29].

Simple examples can be envisaged. For instance, a uniform intensity within the interval, i.e.,

$$
G(\zeta)=G_{0} \operatorname{rect}(\zeta / L)
$$

with constant $G_{0}$, leads to

$$
W_{0}\left(r_{1}, r_{2}\right)=\frac{L G_{0}}{(\lambda f)^{2}} \tau^{*}\left(r_{1}\right) \tau\left(r_{2}\right) \text { sinc }\left[\frac{L\left(r_{2}^{2}-r_{1}^{2}\right)}{2 \lambda f^{2}}\right],
$$

which gives rise to the functional form of the degree of coherence used in the previous numerical examples. The coherence area of the source $\left(\approx \delta_{\mu}^{2}=2 \lambda f^{2} / L\right)$ can be easily modified by changing the focal length of the lens or the length of the line source. As an example, the value $\delta_{\mu}=1 \mathrm{~mm}$ can be obtained with $f=100$ $\mathrm{mm}, L=10 \mathrm{~mm}$ and $\lambda=500 \mathrm{~nm}$.

Summarizing, a new class of sources has been introduced, having the property of being completely coherent for any pair of points on a given ring concentric to the source center. The field is partially coherent for points located at different radial distances from the source center. The geometry of the problem suggests that sources of this kind may find application whenever circular symmetry is required, as, for example, in synthesis procedures of
Bessel beams. A simple optical set up for obtaining the proposed sources has been presented, which uses a primary incoherent line source placed along the axis of the source and exploits an extension of the van Cittert-Zernike theorem, relating the degree of coherence across the source to the line intensity. Our results were originated from symmetry considerations, which also allowed us to identify the class of sources giving rise to fields with the requested coherence characteristics. A posteriori, it could be noticed that such results could have been also derived in the framework of the fields radiated from quasi-homogeneous tridimensional primary sources [29].

Funding. Spanish Ministerio de Economía y Competitividad project FIS2013-46475 and project FIS2016-75147.

\section{REFERENCES}

1. E. Wolf and E. Collett, Optics Communications 25, 293 - 296 (1978).

2. R. Martínez-Herrero and P.M. Mejías, J. Opt. Soc. Am. A 1, 556-558 (1984).

3. F. Gori, G. Guattari, and C. Padovani, Optics Communications 64, 311 316 (1987)

4. R. Simon and N. Mukunda, J. Opt. Soc. Am. A 10, 95-109 (1993).

5. F. Gori, V. Bagini, M. Santarsiero, F. Frezza, G. Schettini, and G.S. Spagnolo, Optical Review 1, 143-145 (1994).

6. S.A. Ponomarenko, J. Opt. Soc. Am. A 18, 150-156 (2001)

7. A.V. Shchegrov and E. Wolf, Opt. Lett. 25, 141-143 (2000)

8. G.V. Bogatyryova, C.V. Fel'de, P.V. Polyanskii, S.A. Ponomarenko, M.S. Soskin, and E. Wolf, Opt. Lett. 28, 878-880 (2003)

9. O. Korotkova, L.C. Andrews, and R.L. Phillips, Opt. Eng. 43, 330-341 (2004)

10. P. Vahimaa and J. Turunen, Opt. Express 14, 1376-1381 (2006).

11. F. Gori, M. Santarsiero, R. Borghi, and C.-F. Li, J. Opt. Soc. Am. A 25, 2826-2832 (2008).

12. R. Martínez-Herrero, P. M. Mejías, and F. Gori, Opt. Lett. 34, 13991401 (2009).

13. H. Lajunen and T. Saastamoinen, Opt. Lett. 36, 4104-4106 (2011).

14. O. Korotkova, S. Sahin, and E. Shchepakina, J. Opt. Soc. Am. A 29, 2159-2164 (2012).

15. Z. Mei and O. Korotkova, Opt. Lett. 38, 2578-2580 (2013).

16. M. Singh, J. Tervo, and J. Turunen, J. Opt. Soc. Am. A 30, 2611-2617 (2013).

17. M. Santarsiero, G. Piquero, J. C. G. de Sande, and F. Gori, Opt. Lett. 39, 1713-1716 (2014).

18. H. Partanen, N. Sharmin, J. Tervo, and J. Turunen, Opt. Express 23, 28718-28727 (2015).

19. Z. Mei and O. Korotkova, Opt. Lett. 40, 2473-2476 (2015).

20. J. C. G. de Sande, M. Santarsiero, G. Piquero, and F. Gori, Journal of Optics 17, 125613 (2015).

21. Y. Chen, S.A. Ponomarenko, and Y. Cai, Applied Physics Letters 7, 39957 (2017).

22. P. De Santis, F. Gori, G. Guattari, and C. Palma, Optics Communications 29, $256-260$ (1979).

23. A.T. Friberg, E. Tervonen, and J. Turunen, J. Opt. Soc. Am. A 11, 1818-1826 (1994).

24. F. Wang and Y. Cai, Opt. Lett. 33, 1795-1797 (2008).

25. Y. Cai, Y. Chen, and F. Wang, J. Opt. Soc. Am. A 31, 2083-2096 (2014).

26. Y. Chen, S.A. Ponomarenko, and Y. Cai, Scientific Reports 109, 10.1063/1.4960966 (2016).

27. C. Liang, F. Wang, X. Liu, Y. Cai, and O. Korotkova, Opt. Lett. 39, 769-772 (2014).

28. G. Gbur and T. Visser, Progress in Optics 55, 285-339 (2010).

29. L. Mandel and E. Wolf, Optical Coherence and Quantum Optics (Cambridge University Press, Cambridge, UK, 1995).

30. M. Abramowitz and I. Stegun, Handbook of Mathematical Functions (Cambridge University Press, Cambridge, UK, 1972)

31. J. Durnin, J. J. Miceli, and J. H. Eberly, Phys. Rev. Lett. 58, 1499-1501 (1987). 


\section{REFERENCES}

1. E. Wolf and E. Collett, "Partially coherent sources which produce the same far-field intensity distribution as a laser," Optics Communications 25, 293 - 296 (1978).

2. R. Martínez-Herrero and P. M. Mejías, "Radiometric definitions for partially coherent sources," J. Opt. Soc. Am. A 1, 556-558 (1984).

3. F. Gori, G. Guattari, and C. Padovani, "Modal expansion for $J_{0}$ correlated sources", Optics Communications 64, 311 - 316 (1987)

4. R. Simon and N. Mukunda, "Twisted Gaussian Schell-model beams", J. Opt. Soc. Am. A 10, 95-109 (1993).

5. F. Gori, V. Bagini, M. Santarsiero, F. Frezza, G. Schettini, and G. S. Spagnolo, "Coherent and partially coherent twisting beams", Optical Review 1, 143-145 (1994).

6. S. A. Ponomarenko, "A class of partially coherent beams carrying optical vortices," J. Opt. Soc. Am. A 18, 150-156 (2001)

7. A. V. Shchegrov and E. Wolf, "Partially coherent conical beams," Opt. Lett. 25, 141-143 (2000)

8. G. V. Bogatyryova, C. V. Fel'de, P. V. Polyanskii, S. A. Ponomarenko, M. S. Soskin, and E. Wolf, "Partially coherent vortex beams with a separable phase," Opt. Lett. 28, 878-880 (2003)

9. O. Korotkova, L. C. Andrews, and R. L. Phillips, "Model for a partially coherent gaussian beam in atmospheric turbulence with application in lasercom," Opt. Eng. 43, 330-341 (2004)

10. P. Vahimaa and J. Turunen, "Finite-elementary-source model for partially coherent radiation," Opt. Express 14, 1376-1381 (2006).

11. F. Gori, M. Santarsiero, R. Borghi, and C.-F. Li, "Partially correlated thin annular sources: the scalar case," J. Opt. Soc. Am. A 25, 2826-2832 (2008).

12. R. Martínez-Herrero, P. M. Mejías, and F. Gori, "Genuine cross-spectral densities and pseudo-modal expansions," Opt. Lett. 34, 1399-1401 (2009).

13. H. Lajunen and T. Saastamoinen, "Propagation characteristics of partially coherent beams with spatially varying correlations," Opt. Lett. 36, 4104-4106 (2011).

14. O. Korotkova, S. Sahin, and E. Shchepakina, "Multi-Gaussian Schellmodel beams," J. Opt. Soc. Am. A 29, 2159-2164 (2012).

15. Z. Mei and O. Korotkova, "Cosine-Gaussian Schell-model sources," Opt. Lett. 38, 2578-2580 (2013).

16. M. Singh, J. Tervo, and J. Turunen, "Elementary-field analysis of partially coherent beam shaping," J. Opt. Soc. Am. A 30, 2611-2617 (2013).

17. M. Santarsiero, G. Piquero, J. C. G. de Sande, and F. Gori, "Difference of cross-spectral densities," Opt. Lett. 39, 1713-1716 (2014).

18. H. Partanen, N. Sharmin, J. Tervo, and J. Turunen, "Specular and antispecular light beams," Opt. Express 23, 28718-28727 (2015).

19. Z. Mei and O. Korotkova, "Alternating series of cross-spectral densities," Opt. Lett. 40, 2473-2476 (2015)

20. J. C. G. de Sande, M. Santarsiero, G. Piquero, and F. Gori, "The subtraction of mutually displaced Gaussian Schell-model beams," Journal of Optics 17, 125613 (2015).

21. Y. Chen, S. A. Ponomarenko, and Y. Cai, "Self-Steering Partially Coherent Beams," Applied Physics Letters 7, 39957 (2017).

22. P. D. Santis, F. Gori, G. Guattari, and C. Palma, "An example of a Collett-Wolf source," Optics Communications 29, 256 - 260 (1979).

23. A. T. Friberg, E. Tervonen, and J. Turunen, "Interpretation and experimental demonstration of twisted Gaussian Schell-model beams", J. Opt. Soc. Am. A 11, 1818-1826 (1994).

24. F. Wang and Y. Cai, "Experimental generation of a partially coherent flat-topped beam," Opt. Lett. 33, 1795-1797 (2008).

25. Y. Cai, Y. Chen, and F. Wang, "Generation and propagation of partially coherent beams with nonconventional correlation functions: a review [invited]," J. Opt. Soc. Am. A 31, 2083-2096 (2014).

26. Y. Chen, S. A. Ponomarenko, and Y. Cai, "Experimental generation of optical coherence lattices," Scientific Reports 109, 10.1063/1.4960966 (2016).

27. C. Liang, F. Wang, X. Liu, Y. Cai, and O. Korotkova, "Experimental generation of cosine-Gaussian-correlated Schell-model beams with rectangular symmetry," Opt. Lett. 39, 769-772 (2014).
28. G. Gbur and T. Visser, "The structure of partially coherent fields," Progress in Optics 55, 285-339 (2010).

29. L. Mandel and E. Wolf, Optical Coherence and Quantum Optics (Cambridge University Press, 1995).

30. M. Abramowitz and I. Stegun, Handbook of Mathematical Functions (Cambridge University Press, Cambridge, UK, 1972)

31. J. Durnin, J. J. Miceli, and J. H. Eberly, "Diffraction-free beams," Phys. Rev. Lett. 58, 1499-1501 (1987). 